\title{
ADVANCEMENT OF AUTOREGRESSIVE CONDITIONAL DURATION MODELS INVOLVING LIQUIDITY AND PRICE DYNAMICS
}

\author{
RASIKA PUSHPAMALI YATIGAMMANA
}

(Received 7 December 2016; first published online 22 March 2017)

2010 Mathematics subject classification: primary 91B24; secondary 62F15, 62G32, 90C31.

Keywords and phrases: autoregressive, conditional duration, liquidity, price dynamics, probit framework, extreme quantiles.

Financial market activity via trade durations and price dynamics are investigated by means of ultra high frequency data. Intertrade arrival times of stock market trades are modelled by an autoregressive conditional duration (ACD) model. Given the distinct features of financial duration data, the conditional distribution of the ACD model has posed challenges for parametric modelling. The problem considered in the thesis revolves around creating a sufficiently flexible parametric distribution to capture tail properties more effectively than similar distributions in the existing ACD literature. Extending the idea, the possibility of adequately modelling the full conditional distribution and better capturing the high near zero density and the heavy tail in financial trade durations, is explored. Furthermore, in terms of price dynamics, enhancing the predictive accuracy of one-step-ahead forecasts while devising a mechanism to propel multi-step-ahead forecasts of stock price movements are probed in detail within an ordered probit framework.

In addressing this multi-faceted problem, an extended version of the Weibull distribution is proposed first, as a new parametric error distribution for the ACD model to counter the existing inadequacy of capturing the tail behaviour of trade durations. The proposed distribution is designed with an extra shape parameter for greater flexibility and its properties are derived. A Bayesian estimator is developed and assessed through a simulation study. Empirical evidence of its performance based on two real-life applications demonstrates robustness. The proposed distribution displays more flexibility $v i s-a$-vis other frequently applied parsimonious distributions, establishing better model fit and forecast accuracy.

Thesis submitted to the University of Sydney in May 2016; degree approved on 8 June 2016; principal supervisor Richard Gerlach, associate supervisors Shelton Peiris and Jennifer Chan.

(c) 2017 Australian Mathematical Publishing Association Inc. 0004-9727/2017 \$16.00 
To better represent the full conditional distribution, a mixture of two simple distributions is developed next, recognising the heterogeneity in trader behaviour. This model is then generalised. A Bayesian estimator is developed and assessed. Based on empirical evidence, the generalised mixture distribution performs best in terms of model fit, while performing well in forecast and risk evaluation. Further, the dynamic nature of trading intensity is aptly captured by the time-varying mixture weights, which perform consistently better than the respective fixed weight counterparts. Forecast and extreme liquidity risk performance are assessed, where measures of extreme liquidity risk are based on extreme quantiles. Recognising the informational role of time on security price formation, the evolution of the price process is modelled via an ordered probit framework, which is employed to predict future price movements. Estimation and statistical inference are based on a maximum likelihood approach, while a Bayesian estimator is developed for comparison. The model discussed provides improved forecast accuracy against an existing study, while providing a benchmark for the 100-step-ahead rolling forecasts. Furthermore, a new dimension is introduced with the aim of producing multi-step-ahead forecasts, where individual explanatory variables are predicted a priori based on their serial correlation structures. This approach enhances flexibility and adaptability of the model to future price changes, targeting risk minimisation.

Chapters 2 and 4 of the thesis are based on results published in the papers [1,2].

\section{References}

[1] R. P. Yatigammana, S. T. B. Choy and J. S. K. Chan, 'Autoregressive conditional duration model with an extended Weibull error distribution', in: Causal Inference in Econometrics, Studies in Computational Intelligence, 622 (Springer International Publishing, Switzerland, 2016), 83-107.

[2] R. P. Yatigammana, M. S. Peiris, R. H. Gerlach and D. Allen, 'Modelling and forecasting stock price movements with serially dependent determinants', submitted.

RASIKA PUSHPAMALI YATIGAMMANA, Discipline of Business Analytics, University of Sydney, New South Wales 2006, Australia e-mail: yatigammanar@ hotmail.com 